\title{
Prevalence and prognosis of unclassifiable interstitial lung disease
}

\author{
Christopher J. Ryerson' ${ }^{1}$, Thomas H. Urbania², Luca Richeldi ${ }^{3}$ Joshua J. Mooney4, \\ Joyce S. Lee ${ }^{4}$, Kirk D. Jones ${ }^{5}$, Brett M. Elicker², Laura L. Koth4, \\ Talmadge E. King Jr ${ }^{4}$, Paul J. Wolters ${ }^{4}$ and Harold R. Collard ${ }^{4}$
}

Affiliations: 'Dept of Medicine, University of British Columbia, Vancouver, BC, Canada. ${ }^{2}$ Dept of Radiology, University of California San Francisco, CA, "Lept of Medicine, University of California San Francisco, CA, and ${ }^{5}$ Dept of Pathology, University of California San Francisco, CA, USA. ${ }^{3}$ Center for Rare Lung Diseases, University of Modena and Reggio Emilia, Modena, Italy.

Correspondence: C.J. Ryerson, St. Paul's Hospital, 1081 Burrard St, Ward 8B, Vancouver, V6Z 1Y6, BC, Canada. E-mail: chris.ryersonahli.ubc.ca

ABSTRACT The aim of this study was to determine the prevalence, characteristics and outcomes of patients with unclassifiable interstitial lung disease (ILD) and to develop a simple method of predicting disease behaviour.

Unclassifiable ILD patients were identified from an ongoing longitudinal cohort. Unclassifiable ILD was diagnosed after a multidisciplinary review did not secure a specific ILD diagnosis. Clinical characteristics and outcomes were compared with idiopathic pulmonary fibrosis (IPF) and non-IPF ILDs. Independent predictors of mortality were determined using Cox proportional-hazards analysis to identify subgroups with distinct disease behaviour.

Unclassifiable ILD was diagnosed in $10 \%$ of the ILD cohort (132 out of 1370 patients). The most common reason for being unclassifiable was missing histopathological assessment due to a high risk of surgical lung biopsy. Demographic and physiological features of unclassifiable ILD were intermediate between IPF and non-IPF disease controls. Unclassifiable ILD had longer survival rates when compared to IPF on adjusted analysis (hazard ratio $0.62, p=0.04$ ) and similar survival compared to non-IPF ILDs (hazard ratio $1.54, \mathrm{p}=0.12$ ). Independent predictors of survival in unclassifiable ILD included diffusion capacity of the lung for carbon monoxide $(\mathrm{p}=0.001)$ and a radiological fibrosis score $(\mathrm{p}=0.02)$.

Unclassifiable ILD represents approximately $10 \%$ of ILD cases and has a heterogeneous clinical course, which can be predicted using clinical and radiological variables.

@ERSpublications

Unclassifiable ILD has a heterogeneous clinical course that can be predicted using clinical and radiological variables http://ow.ly/mdjwg

For editorial comments see page 576 .

This article has supplementary material available from www.erj.ersjournals.com

Received: Aug 222012 | Accepted after revision: Oct 222012 | First published online: Dec 062012

Conflict of interest: Disclosures can be found alongside the online version of this article at www.erj.ersjournals.com

Copyright @ERS 2013 


\section{Introduction}

Proper classification of interstitial lung diseases (ILDs) requires multidisciplinary expertise with input from pulmonologists, thoracic radiologists, and lung pathologists [1-3]. Despite this coordinated effort, some patients cannot be confidently classified with a specific ILD subtype. This can occur when there are nonspecific or conflicting clinical, radiological, or histopathological findings, or when patients are unable or unwilling to undergo diagnostic procedures. These patients are generally labelled as having an "unclassifiable ILD".

The 2002 American Thoracic Society (ATS)/European Respiratory Society (ERS) consensus statement on idiopathic interstitial pneumonias (IIPs) identified unclassifiable ILD as an area requiring further study, but resisted the creation of a formal disease category [2]. Indeed, little is known about the prevalence, characteristics, and outcomes of patients with unclassifiable ILD. The forthcoming ATS/ERS IIP classification update proposes a disease-behaviour classification, which is predominantly based upon expert opinion aimed at providing guidance on the management and prognosis of patients with unclassifiable ILD (document under revision, personal communication; W.D. Travis, Memorial Sloan Kettering Cancer Center, New York, NY, USA). Our main aim was to provide additional, objective, data that would describe the characteristics and outcomes of this population. Specifically, we sought to determine the prevalence of and reasons for unclassifiable ILD, describe the characteristics and outcomes of these patients, and investigate whether there are easily identifiable subgroups of unclassifiable ILD that have distinct and predictable clinical behaviours.

\section{Methods}

\section{Study patients}

The University of California San Francisco (UCSF) ILD clinic is a specialised ILD centre, with most referrals originating from community pulmonologists. All consenting patients are included in the UCSF-ILD database regardless of diagnosis. We identified 1409 ILD patients in the UCSF-ILD database seen between January 2000 and April 2011 (online supplementary fig. E1). Patients with incomplete medical records $(n=39)$ were excluded (online supplementary table E1). These patients had a potentially diagnostic test recommended, but no information was available about whether this test had been performed or the results of this test. Thus the available study population of 1370 patients represents $97.2 \%$ of the overall UCSF-ILD cohort.

Patients were considered to have unclassifiable ILD if a prospective review of clinical, radiological, and pathological data did not reveal a specific diagnosis following multidisciplinary discussion, according to standard criteria where available [1-5]. Patients required fulfilment of established clinical criteria to be confidently diagnosed with idiopathic pulmonary fibrosis (IPF) [3]. For other diagnoses, we required a level of diagnostic certainty that would allow a clinician to confidently label a patient with a given ILD, and move forward with the appropriate treatment for that disease, without further diagnostic evaluation. Patients not meeting these criteria were labelled as unclassifiable ILD. Clinical, radiological, and pathological data of patients with unclassifiable ILD were re-reviewed to identify the reason that the ILD could not be classified as a specific ILD subtype, which included a review of follow-up data. Patients with unclassifiable ILD had up to three suspected diagnoses recorded prospectively during multidisciplinary discussion as a "differential diagnosis" (e.g. IPF versus chronic hypersensitivity pneumonitis (HP) versus connective tissue diseaseassociated ILD (CT-ILD)) [3-5].

Patients with a multidisciplinary diagnosis of IPF, CT-ILD, idiopathic nonspecific interstitial pneumonia (NSIP), or HP were used as ILD controls [3-5]. These control groups were selected because they are the most frequently considered differential diagnoses in our unclassifiable population. CT-ILD, idiopathic NSIP and HP were reported together as a non-IPF ILD control group since baseline characteristics and outcomes were similar among these diagnoses. The UCSF Committee on Human Research approved this project and all patients provided written informed consent.

\section{Measurements}

Baseline data included age, sex, number of pack-years, need for long-term oxygen therapy, prebronchodilator forced vital capacity (FVC), and the diffusing capacity of the lung for carbon monoxide (DLCO) $[6,7]$. The composite physiologic index (CPI) was calculated as previously reported [8]. Follow-up FVC and DLCO were collected at $12 \pm 3$ months, when available. High-resolution computed tomography (HRCT) scans were re-reviewed for this study by an experienced thoracic radiologist, blinded to patient data, for all unclassifiable patients that had a baseline HRCT in the 12 months preceding their initial clinic visit. Radiological variables included: HRCT fibrosis score [9]; presence of a usual interstitial pneumonia (UIP) pattern (UIP, possible UIP, or inconsistent with UIP) [3]; and the presence of honeycombing (yes or no) [10]. Fibrosis score was determined by estimating the per cent of reticular change and honeycombing in 
three regions of each lung, and averaging these scores to produce a total fibrosis score. Date of lung transplantation or death was verified for all patients using database records and the United States Death Registry Index (http://ssdi.rootsweb.ancestry.com/).

\section{Statistical analysis}

Baseline features of unclassifiable-ILD patients were compared pair-wise to IPF and non-IPF controls using a Chi-squared test, t-test, or Wilcoxon rank sum test. Fine-Gray competing-risks regression analysis was used to evaluate the relationship of baseline features with risk of death, treating lung transplantation as a competing risk [11]. Comparison of unclassifiable ILD with control groups was made in a similar manner, including adjustment for age, sex, baseline FVC, and baseline DLCO. Time to death or lung transplantation was illustrated using Kaplan-Meier curves. Assumption of proportional hazards was assessed using Schoenfeld residuals [12]. Disease progression was defined as any of the following within 12 months of the baseline visit: $\geqslant 10 \%$ relative decline in $\mathrm{FVC} ; \geqslant 15 \%$ relative decline in DLCO; lung transplantation; or death. Frequency of disease progression was compared with controls using a Chi-squared test and logistic regression for unadjusted and adjusted analyses, respectively.

Independent time to death predictors were identified using backward selection, forward selection and the Akaike Information Criterion [13]. Predictors with bivariate $\mathrm{p}<0.10$ were evaluated for their independent association with time to death. Predictor variables were transformed to approximate a normal distribution if necessary. Included variables were assessed for co-linearity and interactions. Model accuracy was described using the c-statistic for the prediction of time to death and area under the receiver operating characteristic (AUROC) curve for prediction of disease progression. All data analysis was performed using STATA 11.0 (STATA Corporation, College Station, TX, USA).

\section{Results}

\section{Prevalence and clinical characteristics}

Demographic and clinical characteristics of the 132 patients with unclassifiable ILD (10\% of the total ILD population) and the 538 control patients are shown in table 1 . Patients with unclassifiable ILD had a mean age of 68 years, were equally male and female, and 64\% were former smokers. A baseline HRCT was available for re-review in $83 \%$ of the unclassifiable patients (109 out of 132 patients). Patients with and without an available baseline HRCT had no difference in baseline characteristics. "UIP pattern" on HRCT was present in $17 \%$ of unclassifiable-ILD patients and "possible UIP pattern" was present in 50\%. A slight majority (55\%) of patients with unclassifiable ILD had received treatment for their ILD. The most common treatment was prednisone $(48 \%)$, followed by azathioprine $(9 \%)$.

Patients had five main reasons for being called unclassifiable ILD: provider unwilling to perform a surgical lung biopsy due to high surgical risk (52\%); conflicting clinical, radiological, and histopathological data $(18 \%) ;$ mild/stable disease in which the risk of a biopsy was felt to outweigh the likely benefit $(9 \%)$; insufficient tissue on surgical biopsy (8\%); and unwillingness of patient to undergo a surgical biopsy (8\%). The characteristics of each of these subgroups are shown in the online supplementary table E2. The most

TABLE 1 Characteristics of the unclassifiable ILD and control patients in the study

\begin{tabular}{|c|c|c|c|c|c|}
\hline Variable & Unclassifiable ILD & IPF controls & p-value & Non-IPF controls & p-value \\
\hline Patients n & 132 & 244 & & 294 & \\
\hline Age years & $67.8 \pm 12.9$ & $69.7 \pm 8.8$ & 0.09 & $59.6 \pm 11.9$ & $<0.0005$ \\
\hline \multicolumn{6}{|l|}{ Smoking history } \\
\hline Ever smoked & 63.6 & 75.8 & 0.01 & 46.1 & 0.001 \\
\hline Pack-years & $16.0 \pm 23.9$ & $23.3 \pm 25.2$ & $<0.0005$ & $8.5 \pm 14.9$ & 0.001 \\
\hline Long-term oxygen therapy & 21.9 & 24.7 & 0.54 & 16.8 & 0.26 \\
\hline \multicolumn{6}{|l|}{ Pulmonary function ${ }^{\#}$} \\
\hline FVC $\%$ predicted & $69.0 \pm 22.1$ & $69.1 \pm 17.8$ & 0.97 & $68.7 \pm 20.3$ & 0.89 \\
\hline DLco $\%$ predicted & $47.6 \pm 19.7$ & $44.0 \pm 16.4$ & 0.08 & $48.5 \pm 20.2$ & 0.66 \\
\hline
\end{tabular}

Data are presented as mean \pm SD or \%, unless otherwise stated. ILD: interstitial lung disease; IPF: IPF, idiopathic pulmonary fibrosis; FVC: forced vital capacity; DLCO: diffusing capacity of the lung for carbon monoxide; ${ }^{\#}: n=129$ for unclassifiable patients. The $p$-values are reported for comparison between unclassifiable ILD and each ILD subtype using Chi-squared test (sex, smoking history and oxygen therapy), t-test lage, FVC, DLCo), or Wilcoxon rank sum test (pack-years). 

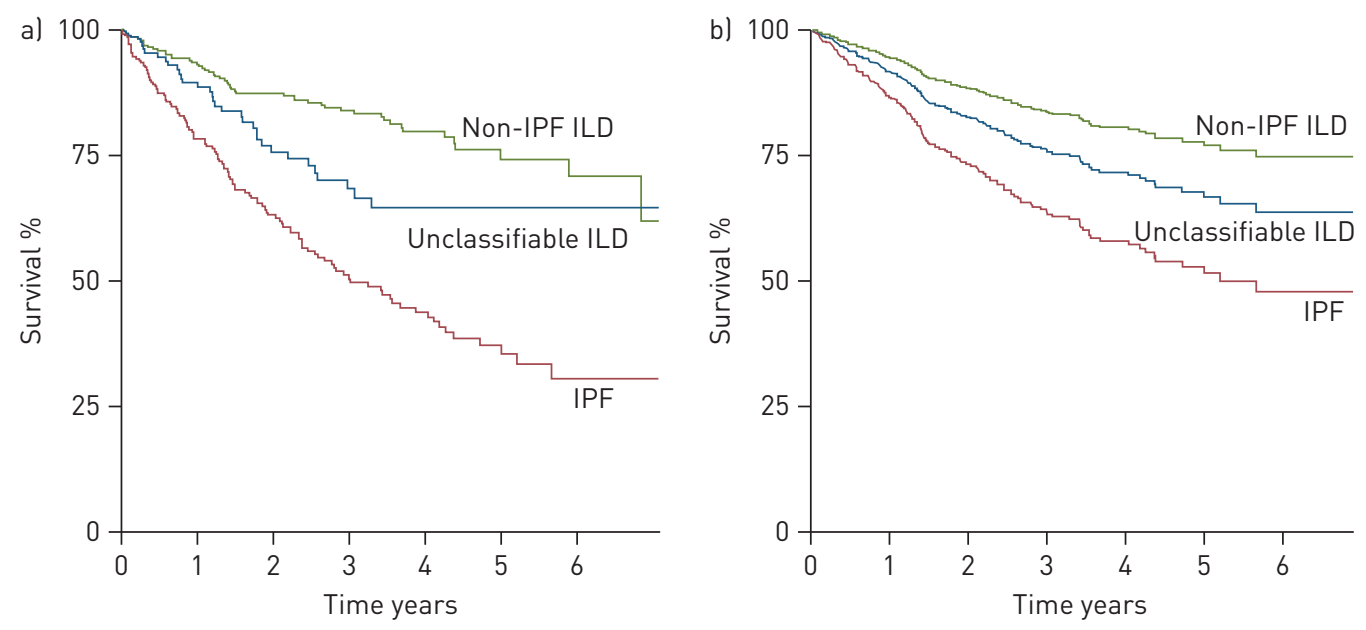

FIGURE 1 Survival analysis (Kaplan-Meier estimates) comparing unclassifiable interstitial lung disease (ILD) to idiopathic pulmonary fibrosis (IPF) and non-IPF ILD controls (connective tissue disease-associated ILD, idiopathic nonspecific interstitial pneumonia or hypersensitivity pneumonitis). a) Shows the unadjusted comparison of unclassifiable ILD with the controls. b) Shows this comparison with adjustment for age, sex, forced vital capacity (FVC), and diffusion capacity of the lung for carbon monoxide (DLCO). Adjusted curves are displayed for the average male patient possessing a mean value for age, FVC, and DL,CO. Unclassifiable ILD had better survival compared to IPF controls (hazard ratio $0.49,95 \%$ CI $0.33-0.72 ; \mathrm{p}<0.0005$ ) and worse survival when compared to non-IPF ILD controls (HR 1.67, 95\% CI 1.07-2.58; $\mathrm{p}=0.02$ ) on unadjusted Cox regression analysis. With adjustment, unclassifiable ILD had better survival when compared to IPF controls (HR 0.62, 95\% CI 0.40-0.97; $\mathrm{p}=0.04$ ), but no difference when compared to non-IPF ILD controls (HR 1.54, 95\% CI 0.89-2.65; $\mathrm{p}=0.12$ ).

TABLE 2 Bivariate clinical and radiological predictors of mortality and disease progression in unclassifiable interstitial lung disease

\begin{tabular}{|c|c|c|c|c|c|}
\hline \multirow[t]{2}{*}{ Variable } & \multirow[t]{2}{*}{ Patients n (\%) } & \multicolumn{2}{|c|}{ Mortality } & \multicolumn{2}{|c|}{ Disease progression ${ }^{\#}$} \\
\hline & & $\operatorname{HR}(95 \% \mathrm{Cl})$ & p-value & OR $(95 \% \mathrm{CI})$ & p-value \\
\hline Sex male \% & & $1.52(0.76-3.08)$ & 0.21 & $1.09(0.40-2.98)$ & 0.87 \\
\hline \multicolumn{6}{|l|}{ Smoking history } \\
\hline Ever smoked \% & & $1.10(0.98-1.24)$ & 0.11 & $1.35(0.48-3.81)$ & 0.57 \\
\hline Long-term oxygen therapy \% & & $2.81(1.35-5.86)$ & 0.006 & $2.19(0.64-7.46)$ & 0.21 \\
\hline Pulmonary function & 129 (98) & & & & \\
\hline FVC \% pred & & $0.85(0.72-1.00)$ & 0.047 & $0.77(0.59-1.00)$ & 0.05 \\
\hline DLCo \% pred & & $0.55(0.42-0.73)$ & $<0.0005$ & $0.66(0.48-0.91)$ & 0.01 \\
\hline Composite physiologic index & & $1.07(1.03-1.10)$ & $<0.0005$ & $1.06(1.01-1.10)$ & 0.01 \\
\hline HRCT findings & 109 (83) & & & & \\
\hline UIP & $19(17)$ & $2.92(0.89-9.61)$ & 0.08 & $4.33(0.80-23.49)$ & 0.09 \\
\hline UIP or possible UIP & $90(83)$ & $2.69(1.00-7.21)$ & 0.049 & $4.73(1.42-15.73)$ & 0.01 \\
\hline Honeycombing \% & $54(50)$ & $2.81(1.21-6.55)$ & 0.02 & $6.57(1.98-21.78)$ & 0.002 \\
\hline \multicolumn{6}{|c|}{$\begin{array}{l}\text { Hazard ratios (HR) and odds ratios (OR) are shown for the bivariate lunadjusted) relationship of each variable with the stated outcome. FVC: forced } \\
\text { vital capacity; DLCO: diffusing capacity of the lung for carbon monoxide; HRCT: high-resolution computed tomography; UIP, usual interstitial } \\
\text { pneumonia. \#: Disease progression was defined as any of the following within } 12 \text { months of the initial visit to the University of California San } \\
\text { Francisco ILD Clinic: } 10 \% \text { decline in FVC, } 15 \% \text { decline in } D \text { LCO, lung transplantation, or death due to any cause (this was only assessable in a } \\
\text { subgroup of patients with complete follow-up data: } n=61) ;{ }^{\uparrow}: H R \text { and OR reported for } 10 \text {-unit change. }{ }^{+} \text {: HR and OR ratios are reported for the } \\
\text { square root of pack-years; }{ }^{\S} \text { : mean } 20.3 \text {. }\end{array}$} \\
\hline
\end{tabular}


TABLE 3 Additional bivariate predictors of mortality and disease progression in unclassifiable interstitial lung disease (ILD): impact of multidisciplinary evaluation and reasons for an unclassifiable diagnosis.

\begin{tabular}{|c|c|c|c|c|c|}
\hline Variable & Patients $\mathbf{n}$ & \multicolumn{2}{|c|}{ Mortality } & \multicolumn{2}{|c|}{ Disease progression" } \\
\hline \multicolumn{6}{|l|}{ Differential diagnoses includes } \\
\hline Hypersensitivity pneumonitis & 91 & $1.13(0.54-2.33)$ & 0.75 & 0.84 (0.28 to 2.53 ) & 0.75 \\
\hline Idiopathic pulmonary fibrosis & 86 & $5.49(1.91-15.74)$ & 0.002 & $4.04(1.28$ to 12.75$)$ & 0.02 \\
\hline Nonspecific interstitial pneumonia & 55 & $1.08(0.55-2.15)$ & 0.82 & $0.97(0.35$ to 2.69$)$ & 0.95 \\
\hline Too old or frail for lung biopsy & 68 & $3.22(1.48-6.97)$ & 0.003 & $2.39(0.85-6.70)$ & 0.10 \\
\hline $\begin{array}{l}\text { Conflicting clinical, radiological and } \\
\text { pathological data }\end{array}$ & 24 & $0.53(0.19-1.51)$ & 0.23 & $0.38(0.12-1.21)$ & 0.10 \\
\hline Mild or stable disease & 12 & $0.25(0.03-1.85)$ & 0.18 & $0.28(0.03-2.85)$ & 0.28 \\
\hline Insufficient tissue on lung biopsy & 11 & $0.46(0.06-3.39)$ & 0.45 & $2.90(0.28-29.53)$ & 0.37 \\
\hline Declined biopsy & 10 & $0.34(0.05-2.52)$ & 0.29 & $1.87(0.16-21.74)$ & 0.62 \\
\hline
\end{tabular}

commonly listed conditions in the differential diagnoses of unclassifiable-ILD patients were HP (68\%), IPF (64\%), NSIP (41\%), CT-ILD (32\%), drug-induced ILD (9\%), and sarcoidosis (8\%).

\section{Outcomes in unclassifiable ILD}

There were 33 deaths and no lung transplantations in the 132 patients with unclassifiable ILD. One-, two-, and five-year mortality rates were $10.6 \%, 23.8 \%$, and $31.1 \%$, respectively (fig. 1). Patients with unclassifiable ILD had significantly longer survival time compared to IPF on unadjusted analysis (hazard ratio $0.49,95 \%$ CI $0.33-0.72 ; \mathrm{p}<0.0005$ ), and with adjustment for age, sex, FVC, and DLCO (HR 0.62, $95 \%$ CI $0.40-0.97 ; p=0.04)$. Unclassifiable ILD had worse survival compared to non-IPF ILD controls on unadjusted analysis (HR 1.67, 95\%CI 1.07-2.58; $\mathrm{p}=0.02$ ), which lost significance on adjusted analysis (HR $1.54,95 \%$ CI $0.89-2.65 ; \mathrm{p}=0.12)$.

Follow-up physiological data was available at 12 months in 61 patients with unclassifiable ILD (46\%). There was no difference in baseline clinical, physiological, or radiological features, when comparing patients with and without a 12-month follow-up. Disease progression occurred in 32 (52\%) unclassifiable-ILD patients, where follow-up data was available, compared to $63 \%$ of patients with IPF $(p=0.16)$ and $45 \%$ of patients with other fibrotic ILDs $(\mathrm{p}=0.38)$. Death in the first year, after baseline assessment, was the most common criteria for disease progression in unclassifiable-ILD patients (41\%), followed by decline in FVC (25\%), decline in both FVC and DLCO (22\%), and DLCO alone (13\%).

Predictors of mortality

Clinical predictors of time to death on bivariate analysis in unclassifiable-ILD patients included: the need for long-term oxygen therapy; baseline FVC; baseline DLCO; the CPI; the provider's unwillingness to perform a surgical lung biopsy, due to high surgical risk; and having a differential diagnosis that included IPF (tables 2 and 3, and fig. 2a). Radiological predictors of time to death on bivariate analysis included HRCT fibrosis score, honeycombing on HRCT, and UIP or possible UIP on HRCT (table 2 and fig. 2b). Multivariate analysis revealed only baseline DLCO and HRCT fibrosis score as independent predictors of time to death, with a c-statistic of 0.81 (table 4). A combination of DLCO (dichotomized at 35\% predicted) and fibrosis score (dichotomized at the median fibrosis score of 20\%) identified patients at low-, intermediate-, and high-risk of mortality (fig. 2c).

\section{Predictors of disease progression}

Predictors of disease progression in unclassifiable ILD patients using bivariate analysis were similar to predictors of time to death (tables 2 and 3). Multivariate analysis revealed only lower baseline DL,CO and higher HRCT fibrosis score as independent predictors of progression (AUROC curve $=0.83$ ) (table 4). 

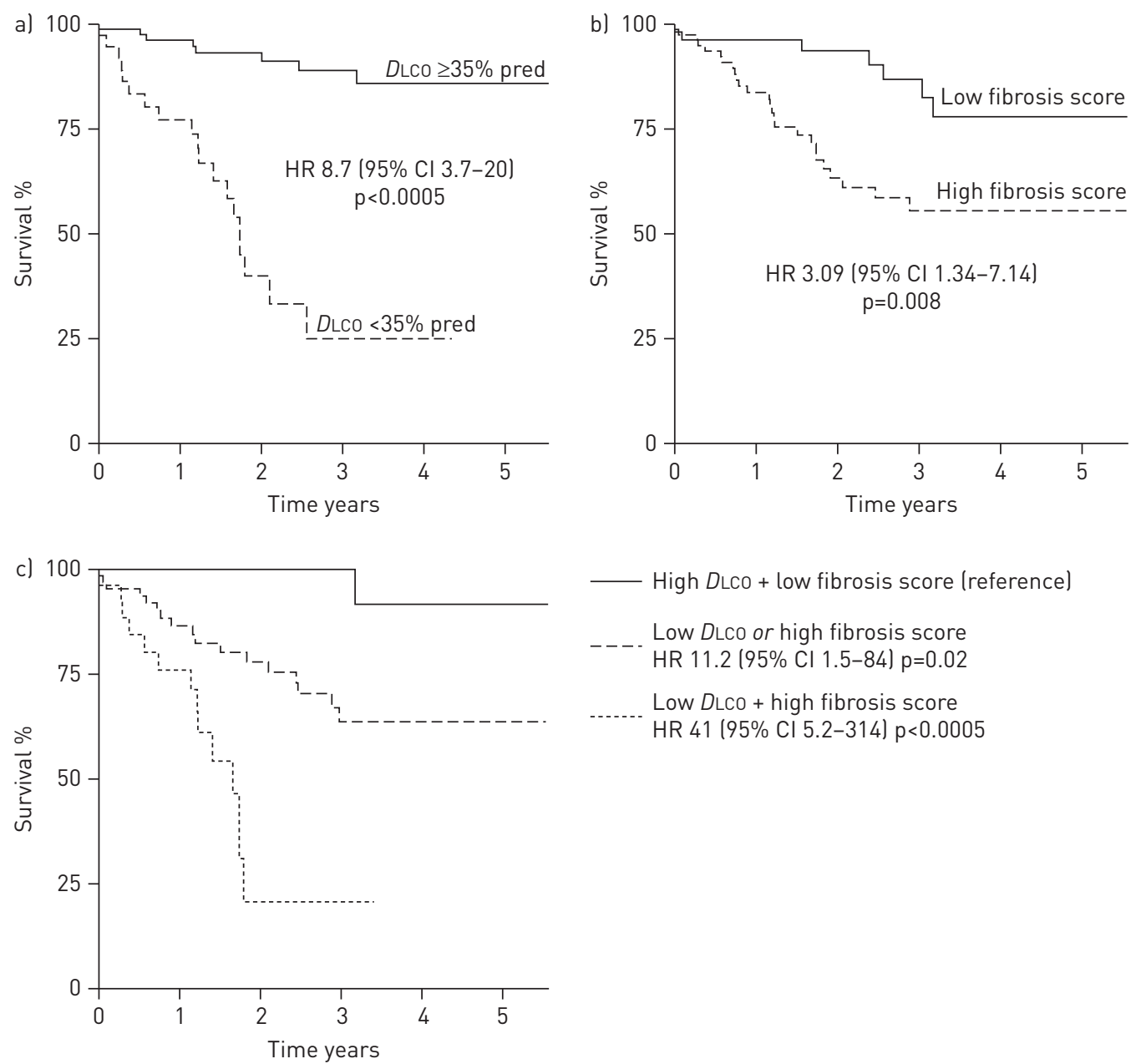

High DLco + low fibrosis score (reference)

Low DLCo or high fibrosis score

HR $11.2(95 \% \mathrm{Cl} 1.5-84) \mathrm{p}=0.02$

Low $D$ LCO + high fibrosis score

HR $41(95 \%$ Cl 5.2-314) p<0.0005

FIGURE 2 Survival of unclassifiable interstitial lung disease (ILD) subgroups. a) Diffusing capacity of the lung for carbon monoxide (DLCO) $\geqslant 35 \%$ pred versus $<35 \%$ pred. b) Median fibrosis score (median fibrosis score $=20 \%$ ). c) Combination of dichotomized DLCO and fibrosis score using the above thresholds. p-values are for the comparison of unclassifiable-ILD subtypes using Cox proportional hazards analysis.

\section{Discussion}

We show in a large, well-described, cohort of patients with ILD that approximately one in 10 patients have an unclassifiable ILD. This makes it the fourth most common classification in our cohort behind IPF (21\%), HP (15\%) and sarcoidosis (14\%). Unclassifiable ILD is associated with clinical characteristics and a prognosis intermediate between IPF and non-IPF ILDs. The risk of disease progression or death in subjects with unclassifiable ILD aligns closely with the presence of baseline clinical and radiological features similar

\section{TABLE 4 Multivariate predictors of mortality and disease progression in unclassifiable interstitial lung disease (ILD)}

\section{Variable}

\section{Mortality}

$\begin{array}{cc}\text { HR }(95 \% \text { CI) } & \text { p-value } \\ 0.59(0.43-0.80) & 0.001 \\ 1.60(1.08-2.37) & 0.02\end{array}$

Disease progression"

\begin{tabular}{cc}
\hline OR $(95 \%$ CI) & p-value \\
\hline $0.67(0.46-0.96)$ & 0.03 \\
$2.29(1.26-4.15)$ & 0.006 \\
\hline
\end{tabular}

Hazard ratios (HR) and odds ratios (OR) are from stepwise regression, using a $p$-value of 0.05 for inclusion. Diffusing capacity of the lung for carbon monoxide (DLCO) and high-resolution computed tomography (HRCT) fibrosis scores were the only variables retained in the multivariate model. \#: disease progression was defined as any of the following within 12 months of the initial visit to the University of California San Francisco ILD Clinic: $10 \%$ decline in forced vital capacity, $15 \%$ decline in DLCO, lung transplantation, or death due to any cause lthis was only assessable in a subgroup of patients with complete follow-up data: $n=61$ ); ${ }^{\top}$ : HR reported for 10 -unit change. 
to IPF, in particular, radiological diagnosis of UIP or possible UIP, HRCT fibrosis score, and the presence of honeycombing.

The 2002 ATS/ERS consensus classification document did not include an unclassifiable ILD disease category because it was felt that this would not be helpful to clinicians [2]. Our finding that $10 \%$ of patients with ILD remain unclassifiable following an extensive multidisciplinary evaluation in an ILD referral centre, argues that unclassifiable ILD is a common problem in a sizable number of patients who still require disease management, and, therefore, is helpful to identify and describe it further.

Unclassifiable ILD is most certainly a heterogeneous collection of ILDs, including IPF and non-IPF conditions, such as chronic HP. Our data suggests that cases with specific clinical features (a low baseline $D \mathrm{~L}, \mathrm{CO}$, high surgical risk precluding lung biopsy, cases in which a diagnosis of IPF is suspected in the differential diagnosis), and with HRCT features suggesting fibrosis (high HRCT fibrosis score, the presence of honeycombing, or the presence of UIP or possible UIP pattern) have a poor prognosis similar to patients with IPF. Whether these cases actually represent patients with IPF, or remain a heterogeneous collection of conditions, is unknown.

The concept of a disease behaviour pattern, in which the ILD phenotype is used to guide management and estimate prognosis, is particularly relevant in unclassifiable ILD, and is a key addition to the forthcoming ATS/ERS IIP classification update. Our results provide additional objective data that support this concept, in particular suggesting that DLCO and HRCT fibrosis score can help guide prognosis in this patient population. Potential management implications of this risk stratification approach (e.g. the use of antifibrotic or anti-inflammatory agents, the timing of lung transplantation evaluation) are beyond the scope of this paper and require further study.

We provide a simple dichotomization of DLCO and fibrosis score to illustrate the importance of these variables and their potential application to clinical practice. We chose a cut-off for DLCO of 35\% predicted for several reasons. First, there are data suggesting that IPF and NSIP have a similarly poor outcome below this threshold [14]. Secondly, surgical lung biopsy has a higher risk of complication at approximately this threshold [15]. Thirdly, this threshold is an appropriate time at which to refer a patient with progressive disease for lung transplantation [16]. Finally, this threshold is commonly used as an enrolment criterion (i.e. $>35 \%$ ) for IPF trials. We stratified fibrosis score at the median value for our cohort, as there is no data to support the use of any specific threshold.

The reported prevalence of unclassifiable ILD may be influenced by this study being performed in an ILD referral centre. In addition, although we followed established criteria for the diagnosis of IPF and other ILDs, our threshold for assigning a diagnosis of unclassifiable ILD may differ from other academic centres. Importantly, inclusion of IPF in the differential diagnosis is dependent on physician expertise, is inherently subjective, and may work differently in less-experienced centres. A baseline HRCT was not available for rereview in $17 \%$ of unclassifiable patients; however, there were no differences in characteristics for those with versus without a baseline HRCT available, suggesting that these missing data did not result in substantial bias. Disease progression data was not available in the majority of study subjects. Baseline characteristics were similar in patients with and without available progression data, and we believe it is unlikely that the lack of follow-up data could lead to a spurious association of DLCO and fibrosis score with disease progression. Furthermore, the consistent association of DLCO and fibrosis score with survival in the complete cohort suggests that the relationship of these variables with disease progression is valid. Finally, we did not have sufficient data to directly evaluate the prevalence or impact of pulmonary hypertension and emphysema in patients with unclassifiable ILD.

In summary, we show that unclassifiable ILD represents $\sim 10 \%$ of ILD cases and has a heterogeneous clinical course that can be predicted using clinical and radiological variables. We believe that unclassifiable ILD is a clinically relevant disease classification for patients with ILD that requires further study. We propose that risk stratification using clinical and radiological features (in particular DLCO and HRCT fibrosis score) may have an important role in the management of patients with unclassifiable ILD. An important area for future research is the evaluation of new and/or improved diagnostic modalities (e.g. serum biomarkers) that can accurately categorise unclassifiable ILD cases into established and distinct disease entities [17-21]. In the absence of serum biomarkers or other biological methods to reclassify unclassifiable-ILD cases, stratification by simple clinical and radiological variables may be useful.

\section{Acknowledgements}

The authors would like to acknowledge the providers and staff of the UCSF Interstitial Lung Disease Program for their assistance in recruiting subjects for this study, the members of the UCSF Interstitial Lung Disease Consortium for their continued referral of patients to our centre, and the patients with ILD who, through their generosity and efforts, allow us to conduct clinical research studies, such as this, in an effort to improve the lives of patients with ILD. 


\section{References}

1 Flaherty KR, King TE Jr, Raghu G, et al. Idiopathic interstitial pneumonia: what is the effect of a multidisciplinary approach to diagnosis? Am J Respir Crit Care Med 2004; 170: 904-910.

2 American Thoracic Society/European Respiratory Society international multidisciplinary consensus classification of the idiopathic interstitial pneumonias. Am J Respir Crit Care Med 2002; 165: 277-304.

3 Raghu G, Collard HR, Egan JJ, et al. An Official ATS/ERS/JRS/ALAT statement: idiopathic pulmonary fibrosis: evidence-based guidelines for diagnosis and management. Am J Respir Crit Care Med 2011; 183: 788-824.

4 Lacasse Y, Selman M, Costabel U, et al. Clinical diagnosis of hypersensitivity pneumonitis. Am J Respir Crit Care Med 2003; 168: 952-958.

5 Travis WD, Hunninghake G, King TE Jr, et al. Idiopathic nonspecific interstitial pneumonia: report of an American Thoracic Society project. Am J Respir Crit Care Med 2008; 177: 1338-1347.

6 Macintyre N, Crapo RO, Viegi G, et al. Standardisation of the single-breath determination of carbon monoxide uptake in the lung. Eur Respir J 2005; 26: 720-735.

Miller MR, Hankinson J, Brusasco V, et al. Standardisation of spirometry. Eur Respir J 2005; 26: 319-338.

8 Wells AU, Desai SR, Rubens MB, et al. Idiopathic pulmonary fibrosis: a composite physiologic index derived from disease extent observed by computed tomography. Am J Respir Crit Care Med 2003; 167: 962-969.

9 Best AC, Meng J, Lynch AM, et al. Idiopathic pulmonary fibrosis: physiologic tests, quantitative CT indexes, and CT visual scores as predictors of mortality. Radiology 2008; 246: 935-940.

10 Hansell DM, Bankier AA, MacMahon H, et al. Fleischner Society: glossary of terms for thoracic imaging. Radiology 2008; 246: 697-722.

11 Fine JP, Gray RJ. A proportional hazards model for the subdistribution of a competing risk. J Am Stat Assoc 1999; 94: 496-509.

12 Grambsch PM, Therneau TM. Proportional hazards tests and diagnostics based on weighted residuals. Biometrika 1994; 81: 515-526.

13 Akaike H. A new look at the statistical model identification. IEEE Trans Automat Contr 1974; 19: 716-723.

14 Latsi PI, du Bois RM, Nicholson AG, et al. Fibrotic idiopathic interstitial pneumonia: the prognostic value of longitudinal functional trends. Am J Respir Crit Care Med 2003; 168: 531-537.

15 Utz JP, Ryu JH, Douglas WW, et al. High short-term mortality following lung biopsy for usual interstitial pneumonia. Eur Respir J 2001; 17: 175-179.

16 Mogulkoc N, Brutsche MH, Bishop PW, et al. Pulmonary function in idiopathic pulmonary fibrosis and referral for lung transplantation. Am J Respir Crit Care Med 2001; 164: 103-108.

17 Ishii $\mathrm{H}$, Mukae $\mathrm{H}$, Kadota J, et al. High serum concentrations of surfactant protein A in usual interstitial pneumonia compared with non-specific interstitial pneumonia. Thorax 2003; 58: 52-57.

18 Greene KE, King TE Jr, Kuroki Y, et al. Serum surfactant proteins-A and -D as biomarkers in idiopathic pulmonary fibrosis. Eur Respir J 2002; 19: 439-446.

19 Ohnishi H, Yokoyama A, Kondo K, et al. Comparative study of KL-6, surfactant protein-A, surfactant protein-D, and monocyte chemoattractant protein-1 as serum markers for interstitial lung diseases. Am J Respir Crit Care Med 2002; 165: 378-381.

20 Casoni GL, Ulivi P, Mercatali L, et al. Increased levels of free circulating DNA in patients with idiopathic pulmonary fibrosis. Int J Biol Markers 2010; 25: 229-235.

21 Rosas IO, Richards TJ, Konishi K, et al. MMP1 and MMP7 as potential peripheral blood biomarkers in idiopathic pulmonary fibrosis. PLoS Med 2008; 5: e93. 